\title{
Drospirenone-containing Oral Contraceptive Pills and the Risk of Venous and Arterial Thrombosis: A Systematic Review
}

Short Title: The Thrombotic Risk of Drospirenone

\author{
Clara Q. Wu ${ }^{1,2}$, Sonia M. Grandi MSc ${ }^{2}$, Kristian B. Filion $\mathrm{PhD}^{2,3}$, Haim A. Abenhaim MD MPH ${ }^{2,4}$, \\ Lawrence Joseph $\mathrm{PhD}^{5,6}$, and Mark J. Eisenberg MD MPH ${ }^{1,2,6,7}$ \\ ${ }^{1}$ Faculty of Medicine, McGill University, Montreal, QC \\ ${ }^{2}$ Center for Clinical Epidemiology, Lady Davis Institute for Medical Research, Jewish General \\ Hospital, Montreal, QC \\ ${ }^{3}$ Division of Clinical Epidemiology, McGill University, Montreal, QC \\ ${ }^{4}$ Department of Obstetrics and Gynecology, Jewish General Hospital, Montreal QC \\ ${ }^{5}$ Division of Clinical Epidemiology, McGill University Health Center, Montreal, QC \\ ${ }^{6}$ Department of Epidemiology, Biostatistics, and Occupational Health, McGill University, Montreal, QC \\ ${ }^{7}$ Division of Cardiology, Jewish General Hospital/McGill University, Montreal, QC
}

\author{
Word Count: 3,06894 \\ Address for Correspondence: \\ Mark J. Eisenberg, MD MPH \\ Professor of Medicine \\ Divisions of Cardiology and Clinical Epidemiology \\ Jewish General Hospital/McGill University \\ 3755 Côte Ste-Catherine Road, Suite H-421.1 \\ Montreal, Quebec, Canada H3T 1E2 \\ Telephone: (514) 340-8222 Ext. 3564 \\ Fax: (514) 340-7564 \\ E-mail: mark.eisenberg@mcgill.ca
}




\section{STRUCTURED ABSTRACT}

Background: Previous studies have provided conflicting results regarding the effect of drospirenone-containing oral contraceptive pills (OCPs) on the risk of venous and arterial thrombosis.

Objectives: To conduct a systematic review to assess the risk of venous thromboembolism (VTE), myocardial infarction (MI), and stroke in individuals taking drospirenone-containing OCPs.

Search Strategy: We systematically searched CINAHL, the Cochrane Library, Dissertation \& Abstracts, EMBASE, HealthStar, Medline, and Science Citation Index from inception to November 2012.

Selection Criteria: We included all case reports, observational studies, and experimental studies assessing the risk of venous and arterial thrombosis of drospirenone-containing OCPs.

Data Collection and Analysis: Data were collected independently by 2 reviewers.

Main Results: A total of 22 studies (6 case reports, 3 case series [including 26 cases], and 13 comparative studies) were included in our systematic review. The 32 identified cases suggest a possible link between drospirenone-containing OCPs and venous and arterial thrombosis. VTE was the primary outcome in all included comparative studies. Incidence rates of VTE among drospirenone-containing OCP users ranged from 23.0 to 136.7 per 100000 woman-years, whereas those among levonorgestrel-containing OCP users ranged from 6.64 to 92.1 per 100000 woman-years. The rate ratio for VTE among drospirenone-containing OCP users ranged from 4.0 to 6.3 compared to non-users of OCPs and from 1.0 to 3.3 compared to levonorgestrelcontaining OCPs users. The arterial effects of drospirenone-containing OCPs were inconclusive. 
Conclusions: Our systematic review suggests that drospirenone-containing OCP use is associated with a higher risk for VTE than no OCP use and levonorgestrel-containing OCP use. 
KEY WORDS: Arterial Thrombosis, Oral Contraceptive Pills, Deep Vein Thrombosis, Drospirenone, Myocardial Infarction, Pulmonary Embolism, Venous Thrombosis. 


\section{INTRODUCTION}

Oral contraceptive pills (OCPs) are associated with an increased risk of thrombotic events. ${ }^{1-3}$ Fourth generation OCPs were introduced to the North American market in $2000 .^{4}$ This new generation is characterized by the addition of the progestin drospirenone, which was believed to be associated with a lower risk of thrombosis. ${ }^{5}$ Drospirenone-containing OCPs are currently the only available oral contraceptive with 3 indications: contraception, the treatment of premenstrual dysphoric disorder, and the treatment of moderate acne. ${ }^{6}$ However, recent observational studies have provided conflicting results regarding the effects of drospirenonecontaining OCPs on the risk of venous thrombosis. ${ }^{7-10}$ In addition, the effect of drospirenonecontaining OCPs on the risk of arterial thrombosis remains controversial. ${ }^{10,11}$ We therefore conducted a systematic review to synthesize the available data regarding drospirenonecontaining OCPs and the risk of venous and arterial thrombotic events, including deep vein thrombosis (DVT), pulmonary embolism (PE), myocardial infarction (MI), and stroke.

\section{METHODS}

\section{Data Sources}

We systematically searched the CINAHL (from 1981 to November 2012), Cochrane Library (from 1898 to November 2012), Dissertation \& Abstracts (from 1861 to November 2012), EMBASE (from 1947 to November 2012), HealthStar (from 1966 to November 2012), Medline (from 1946 to November 2012), and Science Citation Index (from 1900 to November 2012) databases to identify all reports of thrombotic events in women taking OCPs (Appendix S1). OCPs in the current systematic review pertain to estrogen and progestin combination hormonal oral contraceptive pills. Keywords used were levonorgestrel, desogestrel, gestodene, 
norgestimate, and drospirenone. In addition, we searched www.clinicaltrialresults.org for potentially relevant randomized controlled trials (RCTs). We limited our search to studies conducted in the female adult population and reported in English or French. The references of included studies were hand-searched to identify any additional potentially relevant publications.

\section{Inclusion Criteria}

Studies were included if they: 1) were case reports, case series, or comparative studies of women taking drospirenone-containing OCPs; 2) reported at least one of the venous and arterial thrombotic outcomes of interest (DVT, PE, MI, and cerebrovascular events [stroke or transient ischemic attack (TIA)]); and 3) were published in English or French. All studies failing to meet these criteria were excluded.

\section{Data Extraction}

Data from included studies were independently extracted by two reviewers. Disagreements were resolved by consensus or, when necessary, by a third reviewer. Study characteristics such as study design, study period, population, and country of origin were extracted. For each outcome of interest, we extracted incidence rates (IRs) by exposure status and comparative effect measures, including hazards ratios (HRs), odds ratios (ORs), and rate ratios (RRs). Outcome data were extracted with corresponding 95\% confidence intervals (CIs).

We performed this systematic review according to the MOOSE statement since all included studies were observational. ${ }^{12}$ The results of our systematic search are detailed in a flow chart which follows the guidelines outlined by the PRISMA statement (Figure 1). ${ }^{13}$

\section{RESULTS}

\section{Literature Search}


Our search identified 9148 potentially relevant articles (Figure 1). Of these, 9123 were excluded because they were irrelevant to the subject of study $(n=9013)$, editorials or commentaries $(n=62)$, or review articles $(n=48)$. A total of 25 full-text articles were retrieved for further review. Three additional studies were excluded; one presented the rationale and design for a prospective study and the 2 others were subgroup analyses of an already included study. A total of 22 studies (6 case reports, 3 case series [including 26 cases], and 13 comparative studies) were included in our systematic review. No interventional studies met our inclusion criteria.

\section{Case Reports and Case Series}

The 6 case reports and 3 case series contained a total of 32 cases of thrombotic events that occurred in drospirenone-containing OCPs users (Table 1). All reports occurred in patients residing in Europe and were published between 2003 and 2012. A total of 31 patients were taking a combination of $30 \mu \mathrm{g}$ ethinyl estradiol and $30 \mathrm{mg}$ drospirenone; 1 patient was taking $\underline{\mathrm{a}}$ combination of $20 \mu \mathrm{g}$ ethinyl estradiol and $30 \mathrm{mg}$ drospirenone. The median age of patients was 33.5 years (range: 17 to 50 years), and the median duration of drospirenone-containing OCP use before the thrombotic event was 150.5 days (range: 15 to 2557 days). Twenty of the $31 \underline{2}$ patients described in the included case reports and case series had at least one known risk factor for thrombotic disease, including age greater than 35 years, diabetes mellitus, family history of thrombotic disease, hyperlipidemia, hypertension, immobilization, obesity, pregnancy/delivery, smoking, and surgical intervention. Six patients also reported a genetic predisposition for thrombotic disease, having either factor V Leiden mutation, prothrombin G20210A mutation or positive IgG anticardiolipin antibodies. A total of 27 patients experienced VTEs, including 2 reports of venous thrombosis, ${ }^{14,15} 9$ DVTs, ${ }^{14-18} 2$ pulmonary thromboses,${ }^{14,15} 12$ PEs (1 fatal), ${ }^{15,17}$ and 2 of patients with both DVT and PE. ${ }^{15}$ Risk factors were unspecified in 12 of the 27 patients 
with confirmed venous thrombosis. Arterial thrombotic events were reported in 4 patients, 3 of which had an MI, ${ }^{5,19,20}$ and 1 had a TIA. ${ }^{21}$ All 4 patients had at least 1 of 3 risk factors: smoking, family history of MI, and recent surgery.

\section{Comparative Studies and VTE}

A total of 13 comparative studies evaluating the risk of thrombotic events related to the use of drospirenone-containing OCPs were identified (Table 2). Nine of the 13 identified studies were cohort studies, and the remaining 4 were case-control studies. No RCTs were identified. The total patient populations in the individual studies ranged from 867 to 1626158 patients. Studies were reported (either published or included in Food and Drug Administration [FDA] briefing material) between 2007 and 2012 and included data from databases of developed countries, notably the National Registry of Medicinal Products Statistics, National Registry of Patients, Statistics of Denmark, the European Active Surveillance Study (EURAS), German outpatient offices, Ingenix Research Data Mart, the Multiple Environmental and Genetic Assessment study (MEGA), the United Kingdom General Practice Research Database (GPRD), the United States (US) PharMetrics database, Kaiser Permanente Northern California, Kaiser Permanente Southern California, US State Medicaid databases, and the Israeli Clalit Clinical database. The duration of follow-up ranged from 12 to 180 months and occurred from 1995 to 2011. There was heterogeneity in inclusion criteria and user definitions, with 6 studies including prevalent users and 7 involving new users or initiators (Appendix S2).

The primary endpoint of-was VTE for 12 of the included comparative studies (Table 3), and arterial thrombosis for 1 included study (Table 4). Eight studies compared the risk of VTE between drospirenone-containing and levonorgestrel-containing OCP users. The incidence rates for VTE ranged from 23.0 to 136.7 per 100000 women-years (WYs) for drospirenone- 
containing OCP users and from 6.64 to 92.1 per 100000 WYs for levonorgestrel-containing OCP users. Drospirenone-containing OCP users had an increased risk of VTE compared with users of levonorgestrel-containing OCPs, with relative risks ranging from 1.0 to 3.3 . In the 8 studies comparing the risk of VTE between levonorgestrel and drospirenone-containing OCPs, 5 reported a greater risk for VTE among users of drospirenone-containing OCPs, ${ }^{7,8,11,22,23}$ whereas the 3 other studies were inconclusive..$^{9,24,25}$ Two studies examined these associations in both 'all users' and a subgroup of 'new users' of drospirenone-containing OCPs; ${ }^{11,23}$ in both studies, the 'new user' analysis produced results that were consistent with those of the 'all user' analysis with respect to VTE (Table 3).

Two studies investigated the risk of VTE in drospirenone-containing OCP users compared with that in users of other oral contraceptives. ${ }^{26,27}$ One study involved 18 cases of VTE among drospirenone users and 39 among users of other oral contraceptives. The comparison between these different formulations of oral contraceptives was inconclusive due to sparse data $(\mathrm{RR}=0.9,95 \% \mathrm{CI}, 0.5,1.6)$. The other study involved 17 cases of VTE among drospirenone users and 4 among norgestimate and desogestrel users. The authors reported an incidence rate ratio of $6.4 .^{27}$

Three of the included studies compared the risk of VTE in drospirenone-containing OCP users to non-users of OCPs. ${ }^{22,25,28}$ Incidence rate for VTE ranged from 78.3 to 93 per 100000 WYs among drospirenone-containing OCP users and from 37 to 54.7 per 100000 WYs among non-users of OCPs. After adjusting for potential confounders (Appendix S3), drospirenonecontaining OCPs users had a substantially higher risk of VTE (relative risk ranging from 4.0 to 6.3) compared with non-users.

\section{Comparative Studies and Arterial Thrombosis}


Our literature search identified 4 studies that compared the risk of arterial thrombosis between drospirenone-containing and other OCP users (Table 4). Incidence rates for arterial thrombosis ranged from 6.3 to 58 per 100000 WYs among drospirenone-containing OCP users and from 13.2 to 123 per $100000 \mathrm{WYs}$ among levonorgestrel-containing OCP users. In the Long-term Active Surveillance Study (LASS)-stydy ${ }^{10}$ drospirenone-containing OCP users had a substantial reduction in the risk of arterial thrombosis compared with levonorgestrel-containing OCP users $(\mathrm{HR}=0.4 ; 95 \% \mathrm{CI}=0.2,0.9)$ whereas Gronich ${ }^{23}$ and the FDA analysis of all users ${ }^{11}$ produced inconclusive results, with relative risks ranging from 0.81 to 0.87 and the limits of their 95\% CIs including both clinically important harms and benefits. In contrast, when the FDA analysis ${ }^{11}$ was restricted to new users, the HR increased to $1.64(95 \% \mathrm{CI}=0.79,3.40)$, although wide $95 \%$ CIs due to sparse data prevent strong conclusions from being drawn from this analysis.

The comparison of the arterial thrombotic effects of drospirenone-containing OCPs to those of other OCPs also produced heterogeneous results (Table 4). The LASS ${ }^{10}$ found that drospirenone-containing OCPs users had a substantial reduction in arterial thrombosis $(\mathrm{HR}=0.4$, $95 \% \mathrm{CI}=0.2,0.8)$ whereas the FDA's analysis of all-users resulted in an $\mathrm{HR}$ of $0.99(95 \% \mathrm{CI}=$ $0.58,1.69)$. Restriction to new users in the FDA study resulted in an increased risk of arterial thrombosis among drospirenone-containing OCPs users compared with users of other OCPs (HR $=2.01,95 \% \mathrm{CI}=1.06,3.81)$.

The 2012 Lidegaard study compared arterial thrombotic risk between drospirenonecontaining OCPs users to non-users of $\mathrm{OCPs}^{29}$. In this study, drospirenone-containing OCPs were associated with an increased risk of stroke $(\mathrm{RR}=1.64,95 \% \mathrm{CI}=1.24,2.18)$ and $\mathrm{MI}(\mathrm{RR}=$ $1.65,95 \% \mathrm{CI}=1.03,2.63)$. 


\section{DISCUSSION}

\section{Main Findings}

Our study was designed to summarize the available evidence regarding the venous and arterial thrombotic risk of drospirenone-containing OCPs. The evidence to date suggests that drospirenone-containing OCPs may increase the risk of VTE compared with levonorgestrelcontaining OCPs and non-use of OCPs. The effects of drospirenone-containing OCPs on the risk of arterial thrombosis remain unclear with studies included in this review providing conflicting results with some suggesting a protective effect ${ }^{23}$ and others suggesting a doubling of risk. ${ }^{11}$

Twenty out of the 32 cases identified in case reports and case series had at least one concomitant risk factor for thrombotic events, highlighting the need to screen for thrombotic risk factors before initiating OCPs. Furthermore, although duration of OCP use varied among cases, the majority of thrombotic events occurred during the first year of OCP use (28 out of 32 cases). Comparative studies involving patients initiating OCP therapy ${ }^{24,26}$ also had greater incidence rates for VTE than those involving prevalent OCP users. ${ }^{7,8}$ This trend is consistent with conclusions drawn from previous studies investigating thrombotic risk with the use of second and third generation OCPs. ${ }^{25}$

\section{Interpretation in Light of Previous Studies}

The elevated VTE risk that occurs following the initiation of OCP use has important implications for the design and analysis of observational studies of this association. With the greatest risk occurring following the initiation of therapy among first time users, the failure to

properly account for history of OCP use may result in spurious findings. ${ }^{30,31}$ In addition, the inclusion of prevalent or current users may result in an important underestimation of treatment effects since those who experienced events early after the initiation of therapy (but before the 
study period) are excluded for having a history of thrombosis. ${ }^{32}$ User definitions utilized in the included studies varied (Appendix S3), which may explain some of the observed heterogeneity of results. For example, the FDA-funded study's restriction to new users of drospirenonecontaining OCPs resulted in substantially higher risks of arterial thrombosis. Moreover, the estrogen dose, though known to be associated with higher risk of both venous and arterial thrombosis, ${ }^{25,29}$ was unspecified in several of the included studies. These potential methodological limitations of included studies need to be considered when weighing the strength of the evidence supporting the association between drospirenone-containing OCPs and thrombotic risk.

Importantly, while drospirenone-containing OCPs appear to increase the risk of VTE and have unclear effects on the risk of arterial thrombosis, the absolute risk of thrombosis when using these agents remains low. Among drospirenone-containing OCP users, the incidence rate ranged from 23.0 to 136.7 per $100000 \mathrm{WYs}$ for VTE and 6.3 to 58 per $100000 \mathrm{WYs}$ for arterial thrombosis. Hence, there is likely insufficient evidence to recommend discontinuing use of drospirenone-containing OCPs, particularly among long-term users. However, women with VTE are also at risk for developing arterial thrombotic events ${ }^{33}$, and patients should be provided with our current understanding of the risks and benefits associated with the use of these agents to allow for informed decision-making.

In 2011, the Medicines and Healthcare products Regulatory Agency (MHRA) in the United Kingdom, the US FDA, and Health Canada conducted reviews concluding that drospirenone-containing OCPs may be associated with a 1.5 to 3 times higher risk of VTE, and warning labels have been revised to adequately reflect this risk. ${ }^{11,34-36}$ These results are supported by the findings of our systematic review. It should be noted that the statements 
released by these regulatory agencies dealt only with venous effects and that the arterial effects of drospirenone-containing OCP remain under-investigated.

\section{Strengths and Limitations}

Our systematic review was the first to evaluate the safety of drospirenone-containing OCPs with respect to both venous and arterial thrombotic outcomes. The inclusion of detailed case reports allows for a clinically-relevant examination of thrombotic risk factors among exposed cases while the inclusion of comparative studies allows for rigorous statistical adjustment for potential confounders and uses a comparison group to account for the underlying thrombotic risk in this population. The effect of OCPs, including that of drospirenone-containing OCPs, on the risk of venous thrombosis was recently examined in two systematic reviews and meta-analyses. ${ }^{37,38}$ However, the literature searches for these two previous reviews were conducted in April-May 2010, and nine studies have since been completed. Furthermore, given the heterogeneity across studies, the meta-analysis of these data is questionable.

Our study has several potential limitations. First, due to the heterogeneity of comparators, user definitions, and effect measures reported, we were unable to pool data across studies to derive a single overall summary estimate. Secondly, our systematic search did not identify any interventional studies examining this issue. Given the observational nature of the included studies, there is the possibility of confounding by indication. ${ }^{39}$ In addition, based on the antimineralocorticoid and anti-androgenic properties of drospirenone, OCPs containing this progestin may have been preferentially prescribed to women with conditions associated with a higher risk of VTE and arterial thrombosis. ${ }^{40}$ Furthermore, despite the use of rigorous statistical adjustment (Appendix S2), the possibility of residual confounding remains. All included studies contain various degrees of switching between OCPs, and the inadequate adjustment for prior use 
will likely over-estimate the risk of VTEthrombosis. In addition, the present systematic review was limited to studies published in English or French and thus may be affected by language bias. There is widespread awareness of the association between VTE, which is often asymptomatic, and OCP use. ${ }^{40}$ Thus, included studies may be affected by detection bias. ${ }^{41}$

\section{Conclusions}

Although studies examining the thrombotic effects of drospirenone-containing OCPs have methodological limitations, our systematic review suggests that users of these oral contraceptives may be at greater risk for VTE than either non-users of OCPs or users of levonorgestrel-containing OCPs. Despite the observed increased VTE risk, the absolute risk of thrombosis remains low. Physicians should therefore consider the indication for use and the risk-benefit profile of the individual patient prior to prescribing these OCPs. With available studies providing conflicting results, the effect of drospirenone-containing OCPs on arterial thrombosis remains unclear. Further studies on the arterial thrombotic effects of these OCPs are warranted.

\section{ACKNOWLEDGEMENTS}

We would like to thank Renee Atallah, Tara Dourian, Caroline Franck, and Elana Folbe for their help with data abstraction.

\section{DISCLOSURE OF INTERESTS}

The authors have no relationships to disclose.

\section{CONTRIBUTION TO AUTHORSHIP}


CQW conducted the literature search, extracted data, and drafted the manuscript. All authors contributed to study design, interpretation of data, and critically reviewed the manuscript for important intellectual content.

\section{DETAILS OF ETHICS APPROVAL}

This study involved published data and so did not require ethics approval.

\section{FUNDING}

This study was funded by the Canadian Institutes of Health Research (CIHR - grant number MOP-119362). CQW is supported by the Mach-Gaensslen Foundation of Canada Student Grant, funded through the McGill Research Bursary Program. KBF is a CIHR New Investigator. 


\section{REFERENCES}

1. Tchaikovski SN, Rosing J. Mechanisms of estrogen-induced venous thromboembolism. Thrombosis Research 2010;126:5-11.

2. Tanis BC, van den Bosch MA, Kemmeren JM, et al. Oral contraceptives and the risk of myocardial infarction. New England Journal of Medicine 2001;345:1787-93.

3. Vandenbroucke JP, Rosing J, Bloemenkamp KW, et al. Oral contraceptives and the risk of venous thrombosis. New England Journal of Medicine 2001;344:1527-35.

4. Petitti DB. Combination estrogen-progestin oral contraceptives. New England Journal of Medicine 2003;349:1443-50.

5. Orti G, Mira Y, Vaya A. Acute myocardial infarction associated with Yasmin oral contraceptive. Clinical and Applied Thrombosis/Hemostasis 2007;13:336-7.

6. Mishell DR, Jr. YAZ and the novel progestin drospirenone. The Journal of reproductive medicine 2008;53:721-8.

7. Parkin L, Sharples K, Hernandez RK, Jick SS. Risk of venous thromboembolism in users of oral contraceptives containing drospirenone or levonorgestrel: nested casecontrol study based on UK General Practice Research Database. British Medical Journal $2011 ; 342$.

8. Jick SS, Hernandez RK. Risk of non-fatal venous thromboembolism in women using oral contraceptives containing drospirenone compared with women using oral contraceptives containing levonorgestrel: case-control study using United States claims data. British Medical Journal 2011;342:d2151.

9. Dinger J, Assmann A, Mohner S, Minh TD. Risk of venous thromboembolism and the use of dienogest- and drospirenone-containing oral contraceptives: Results from a 
German case-control study. Journal of Family Planning and Reproductive Health Care 2010;36:123-9.

10. FDA Advisory Committee Joint Meeting: Reproductive Health Drugs; Drug Safety and Risk Management. 2011. (Accessed January 12, 2012, at http://www.fda.gov/downloads/AdvisoryCommittees/CommitteesMeetingMaterials/Dru gs/ReproductiveHealthDrugsAdvisoryCommittee/UCM284784.pdf.)

11. FDA Drug Safety Communication: Updated information about the FDA-funded study on risk of blood clots in women taking birth control pills containing drospirenone. 2011. $\begin{array}{llll}\text { (Accessed } & \text { December } & 30, & \text { at }\end{array}$ http://www.fda.gov/Drugs/DrugSafety/ucm299305.htm.)

12. Stroup DF, Berlin JA, Morton SC, et al. Meta-analysis of observational studies in epidemiology: A proposal for reporting. Journal of the American Medical Association 2000;283:2008-12.

13. Moher D, Liberati A, Tetzlaff J, Altman DG. Preferred reporting items for systematic reviews and meta-analyses: the PRISMA statement. Ann Intern Med 2009;151:264-9, W64.

14. Van Grootheest K, Vrieling T. Drug points: Thromboembolism associated with the new contraceptive Yasmin. British Medical Journal 2003;326:257.

15. Lopez M, Vaya A, Martinez Triguero ML, et al. Yasmin and venous thromboembolism: New case reports. Clinical Hemorheology and Microcirculation 2009;42:65-9.

16. Oo TH. Deep vein thrombosis associated with the new oral contraceptive Yasmin. Southern Medical Journal 2009;102:985-6. 
17. Pearce HM, Layton D, Wilton LV, Shakir SAW. Deep vein thrombosis and pulmonary embolism reported in the Prescription Event Monitoring Study of Yasmin. British Journal of Clinical Pharmacology 2005;60:98-102.

18. Marti Gil C, Mulet Alberola A, Mejia Recuero M, Barreira Hernandez D, Llopis Salvia P, Barreda Hernandez D. Update on combined oral contraceptives: Risk of venous thromboembolism. Drospirenone versus other progestins. European Journal of Hospital Pharmacy: Science and Practice 2012;19 (2):254.

19. Cabou C, Lacroix I, Roncalli J, et al. Myocardial infarction in a young female smoker taking oral contraception. Infarctus du myocarde chez une jeune femme fumeuse sous contraception orale. Archives des Maladies du Coeur et des Vaisseaux 2006;99:80-5.

20. Zehir R, Karabay CY, Kocabay G. Myocardial infarction and spontaneous dissection of coronary artery due to oral contraceptive. Journal of Cardiovascular Medicine 2011;12:448-50.

21. Vaya A, Mira Y, Ferrando F, Aznar J, Garcia-Fuster Ma J. Transient ischaemic attack associated with the new contraceptive Yasmin. Thrombosis Research 2004;112:121.

22. Lidegaard O, Nielsen LH, Skovlund CW, Skjeldestad FE, Lokkegaard E. Risk of venous thromboembolism from use of oral contraceptives containing different progestogens and oestrogen doses: Danish cohort study, 2001-9. British Medical Journal 2011;343:d6423.

23. Gronich N, Lavi I, Rennert G. Higher risk of venous thrombosis associated with drospirenone-containing oral contraceptives: A population-based cohort study. Canadian Medical Association Journal 2011;183:E1319-E25. 
24. Dinger JC, Heinemann LA, Kuhl-Habich D. The safety of a drospirenone-containing oral contraceptive: final results from the European Active Surveillance Study on oral contraceptives based on 142,475 women-years of observation. Contraception 2007; 75:344-54.

25. Lidegaard O, Lokkegaard E, Svendsen AL, Agger C. Hormonal contraception and risk of venous thromboembolism: national follow-up study. British Medical Journal 2009;339:b2890.

26. Seeger JD, Loughlin J, Eng PM, Clifford CR, Cutone J, Walker AM. Risk of thromboembolism in women taking ethinylestradiol/drospirenone and other oral contraceptives. Obstetrics and Gynecology 2007;110:587-93.

27. Leppee M, Culig J. Difference between drospirenone-containing oral contraceptives and other oral contraceptives related to risk of venous thromboembolism. Journal of Family Planning and Reproductive Health Care 2012;38:137-8.

28. Vlieg AV, Helmerhorst FM, Vandenbroucke JP, Doggen CJM, Rosendaal FR. The venous thrombotic risk of oral contraceptives, effects of oestrogen dose and progestogen type: results of the MEGA case-control study. British Medical Journal 2009;339.

29. Lidegaard O, Lokkegaard E, Jensen A, Skovlund CW, Keiding N. Thrombotic stroke and myocardial infarction with hormonal contraception. New England Journal of Medicine 2012;366:2257-66.

30. Suissa S. The Transnational study of oral contraceptive cardiovascular safety: history and science. Journal of Clinical Epidemiology 2009;62:588-93. 
31. Suissa S, Blais L, Spitzer WO, Cusson J, Lewis M, Heinemann L. First-time use of newer oral contraceptives and the risk of venous thromboembolism. Contraception 1997;56:141-6.

32. Ray WA. Evaluating Medication Effects Outside of Clinical Trials: New-User Designs. American Journal of Epidemiology 2003;158:915-20.

33. Sorensen HT, Horvath-Puho E, Pedersen L, Baron JA, Prandoni P. Venous thromboembolism and subsequent hospitalisation due to acute arterial cardiovascular events: a 20-year cohort study. Lancet 2007;370:1773-9.

34. Yasmin: risk of venous thromboembolism higher than levonorgestrel-containing pills. 2011. (Accessed December 30, 2011, at http://www.mhra.gov.uk/Safetyinformation/DrugSafetyUpdate/CON120214.)

35. Yasmin and Yaz (drospirenone): Updated information on increased risk of blood clots. 2011. (Accessed December 30, 2011, at http://www.hc-sc.gc.ca/ahcasc/media/advisories-avis/_2011/2011_164-eng.php.)

36. FDA Drug Safety Communication: Updated information about the risk of blood clots in women taking birth control pills containing drospirenone. 2012. (Accessed May 14, 2012, at http://www.fda.gov/Drugs/DrugSafety/ucm299305.htm.)

37. Manzoli L, De Vito C, Marzuillo C, Boccia A, Villari P. Oral contraceptives and venous thromboembolism: a systematic review and meta-analysis. Drug Safety 2012;35:191205.

38. Martinez F, Ramirez I, Perez-Campos E, Latorre K, Lete I. Venous and pulmonary thromboembolism and combined hormonal contraceptives. Systematic review and metaanalysis. Eur J Contracept Reprod Health Care 2012;17:7-29. 
39. Lidegaard $\mathrm{O}$. The influence of thrombotic risk factors when oral contraceptives are prescribed. A control-only study. Acta Obstetricia et Gynecologica Scandinavica 1997;76:252-60.

40. Heinemann LA, Dinger J. Safety of a new oral contraceptive containing drospirenone. Drug Safety 2004;27:1001-18.

41. Dinger J, Shapiro S. Combined oral contraceptives, venous thromboembolism, and the problem of interpreting large but incomplete datasets. Journal of Family Planning \& Reproductive Health Care 2012;38:2-6. 


\section{FIGURE LEGEND}

Figure 1. PRISMA flow diagram of systematic literature search 